\title{
Application du programme leader selon les principes de base du développement local
}

Implementation of the Program Leader against the principe of principles of local development

\section{Pascal Chevalier and Marc Dedeire}

\section{(2) OpenEdition}

\section{Journals}

Electronic version

URL: http://journals.openedition.org/economierurale/4382

DOI: $10.4000 /$ economierurale. 4382

ISSN: 2105-2581

\section{Publisher}

Société Française d'Économie Rurale (SFER)

Printed version

Date of publication: 15 July 2014

Number of pages: 9-25

ISSN: 0013-0559

\section{Electronic reference}

Pascal Chevalier et Marc Dedeire, «Application du programme leader selon les principes de base du développement local », Économie rurale [En ligne], 342 | juillet-août 2014, mis en ligne le 15 juillet 2016, consulté le 01 mai 2019. URL : http://journals.openedition.org/economierurale/4382 ; DOI : 10.4000/ economierurale.4382 


\title{
Application du programme Leader selon les principes de base du développement local
}

\author{
Pascal CHEVALIER • Marc DEDEIRE • Université Montpellier 3, Laboratoire ART-Dev, Montpellier \\ pascal.chevalier@univ-montp3.fr ; marc.dedeire@univ-montp3.fr
}

Le programme de développement local Leader n'est pas un outil clé en main appliqué indifféremment dans chaque pays de I'Union européenne. Si les dispositifs définis au niveau européen y sont applicables selon des modalités identiques, chaque État en décline les objectifs en fonction des priorités qu'il se fixe. L'État intègre le programme dans sa propre stratégie de développement rural. De cette interprétation découlent des formes différenciées de mise en œuvre nationale de la politique européenne qui peuvent avoir un impact sur l'efficacité de la programmation Leader. L'objectif de cet article est d'analyser les rapports entre les interprétations nationales du programme européen et les principes de base du développement local. À partir d'analyses statistique et cartographique, sont élaborées des typologies nationales à l'échelle européenne qui confrontent les modalités de mise en œuvre du programme Leader dans chaque pays avec les principes fondamentaux du développement local.

MOTS-CLÉS : rural, développement local, politique publique, Europe, typologies

\section{Implementation of the Program Leader against the principe of principles of local development}

The Local development program LEADER is not a turnkey tool equally applied in each EU country. Despite the fact that the local development framework defined at the European Union level is applicable according to the same procedures, each country assumes their aims according to their own priorities. EU Countries integrate the program into their own strategy for rural development. Resulting differentiated forms of national implementations of EU policy may impact the effectiveness of the LEADER program. The focus of this paper is to analyze the relationship between national interpretations of the European program and the baselines of local development. With mapping and statistical analyzes, this article proposes national typologies at European level that confront the terms of implementation of the LEADER program in each country with the main principles of local development. (JEL: O210).

KEYWORDS: rural, local development, public policy, Europe, typologies

$\mathbf{L}^{\mathrm{s}}$ 'intégration à l'Union européenne (UE) crée des conditions favorables à la circulation des idées, des modèles et des instruments politiques. Parmi ceux-ci, la démarche de l'action publique se référant au paradigme du « développement local» s'est progressivement imposée à l'échelle du continent. Voulant « légitimer politiquement et institutionnellement le local au sein de l'Europe » (Smith, 1995), cette démarche a instauré de nouveaux dispositifs d'action publique s'organisant selon une conception du territoire voulant accorder plus de place à la répartition des responsabilités entre les acteurs institutionnels et la société civile locale, à l'expérimentation de nouveaux modes de gestion territoriale locale et à la négociation entre les acteurs situés aux différentes échelles de gouvernance infra- et supranationale. Portée par l'UE, cette démarche présente comme emblème le programme Leader, créé en 1991. Fondée sur une logique ascendante de l'action locale (bottom-up), elle doit participer de cette dynamique qui consiste en théorie à promouvoir le développement 
socio-économique des zones rurales par le « bas » (Maurel, 2008). Les initiatives locales sont organisées par des partenariats entre acteurs et sont encadrées par des procédures de contractualisation avec les différents échelons du système territorial. Approche intégrée du développement sur une base territoriale, cette démarche veut également orienter les efforts de développement sur des projets conçus à l'échelle des communautés rurales dans le but de renforcer la capacité d'initiative des acteurs locaux. Elle souhaite enfin participer à l'émergence d'un nouveau mode de gouvernance territoriale qui tend à vouloir renforcer «la démocratie locale et participative » (Dolowitz, Marsh, 2000).

Néanmoins, le second pilier de la PAC, et a fortiori le programme de développement local Leader, ne sont pas des outils de développement clé en main appliqués indifféremment dans chaque pays de l'UE. Si les dispositifs définis au niveau européen y sont applicables selon des modalités identiques, chaque État en décline les objectifs en fonction des priorités qu'il se fixe. Il dispose également d'une grande latitude concernant les moyens financiers qu'il attribue au programme et une liberté dans les modalités de son application au travers de sa propre politique nationale et des outils juridiques et réglementaires qu'il met en place. De fait, il interprète la politique européenne qu'il traduit sous forme de programmes intégrés à sa propre stratégie de développement rural. De cette interprétation découlent des formes différenciées de mise en œuvre nationale de la politique européenne qui peuvent avoir un impact sur l'efficacité et/ou l'efficience de la programmation Leader dans chaque pays de l'UE. Dès lors, il est question, dans cet article, d'analyser les rapports entre la traduction «sur le terrain » des interprétations nationales du programme européen et « la philosophie sur le développement local » que s'efforce d'impulser l'UE et qui constituerait, selon elle, une base nécessaire pour le développement rural. Les modalités de mise en œuvre nationale du programme Leader et leur traduction dans l'application et le fonctionnement des territoires de projets sont-elles en adéquation avec les principes européens sur le développement local ? Peuvent-elles favoriser un développement rural endogène et durable comme le prône l'UE ?

Cet article est organisé en trois parties. Dans une première partie, il est question de revenir sur les principes mêmes du développement local qui sont définis par l'UE et qui doivent, en théorie, s'appliquer à l'ensemble des États membres (Bulmer, Dolowitz, Humphereys, Padgett, 2007). Nous reviendrons particulièrement sur son assise théorique et ses modalités de transfert à chaque État membre (Delpeuch, 2009). Ensuite, nous analyserons les traductions spatiales, dans les 27 pays de l'UE, des modalités d'application de cette politique européenne en matière de constitution des territoires de projet. Elle se fera à partir de la réalisation de typologies issues de l'exploitation statistique des maquettes budgétaires nationales de 2012. Construites à partir des caractéristiques des territoires locaux Leader (en matière de périmètres et de maillage) et repositionnées dans les cadres administratif et juridique propres à chaque pays, ces typologies permettent de voir que, outre les réalités locales (dont il n'est pas question dans cet article, car méritant un travail empirique plus important), elles reflètent avant tout des contextes institutionnels qui peuvent, sous certains aspects, entraver l'efficacité du programme. Enfin, nous confronterons le discours européen sur le développement local à sa traduction effective dans les États. Les types de territoires de projets créés, les modalités de leur sélection et de leur fonctionnement sont-ils conformes au discours dominant que s'efforce d'impulser l'UE? 


\section{Du modèle européen de développement local à l'instrument Leader}

Une grande majorité de chercheurs s'accordent à dire que le développement local serait avant tout une dynamique économique et sociale, voire culturelle, plus ou moins concertée, impulsée par des acteurs individuels et collectifs sur un territoire donné (Teisserenc, 1994 ; Houée, 2001 ; Pecqueur, 2000 ; Greffe, 2005 ; etc.). On pourrait le définir comme un processus qui permettrait de faire mûrir des priorités, de choisir des actions à partir de savoirs et de propositions de groupes de populations habitant un territoire donné, et de mettre en œuvre les ressources disponibles pour satisfaire ces propositions. Le développement local inciterait à privilégier les acteurs plus que les infrastructures, les réseaux plus que les institutions établies, pour donner aux hommes et aux groupes directement intéressés une fonction de décision sur les actions qu'ils mènent. Il ressort de cette approche quelques hypothèses fortes. Le développement local serait d'abord un processus décisionnel. Le territoire de référence pour soutenir une dynamique de développement local ne serait pas isolé, mais articulé à des espaces plus vastes. Les ressources disponibles, techniques, financières et humaines seraient bien évidemment prioritairement celles les plus accessibles dans ce territoire, mais aussi celles acquises de l'extérieur. Il ne s'agirait donc pas de rechercher une quelconque autarcie illusoire ni de faire supporter au niveau local des charges qu'il ne peut assumer. Mieux, le développement local trouverait sa pleine expression avec la mobilisation d'acteurs locaux prenant en compte le contexte national au sein duquel ils agissent. La dimension territoriale, à savoir un espace géographiquement limité et déterminé, constituerait une référence forte du développement local, mais non une exclusivité. Cet espace serait à considérer aussi dans sa dimension sociale. Le sentiment d'appartenance serait un facteur essentiel, d'autant plus indispensable que l'espace géographique n'est pas homogène. Il n'y aurait pas de développement local sans existence d'une communauté territoriale (Buller, 2000).

C'est dans les pays d'Europe occidentale que ces principes du modèle de développement local, en tant qu'action publique, ont pris naissance (Baudelle, Mérenne-Schoumaker, 2011). Dans la plupart des anciens États de l'UE, la nécessité d'appréhender les problèmes de développement rural dans un cadre local a été ressentie dès la fin des années 1960, notamment au Royaume-Uni et en France. Elle s'est concrétisée dans les années 1970 par la mise en place d'outils nationaux (Plans d'aménagement rural en France, Schémas de développement régionaux au Royaume-Uni, etc.) gérés principalement par les ministères de l'Agriculture. Leurs objectifs étaient d'élaborer, sur des périmètres restreints, mais supposés cohérents par les autorités nationales, des programmes de développement multisectoriel qui devaient permettre de coordonner les diverses interventions publiques pour qu'elles concourent à un aménagement global. Avec plus ou moins de vigueur selon les États, et en fonction de l'intensité de fragmentation de la trame communale (qui n'offre alors ni les moyens financiers ni les capacités suffisantes aux communes pour mettre en œuvre des projets), l'intercommunalité s'organise progressivement. D'abord dans les pays anglo-saxons, elle s'étend ensuite à des degrés divers dans la plupart des États ; intercommunalité de gestion axée dans un premier temps sur la réalisation d'équipements ou de services communs, elle s'élargit progressivement à une intercommunalité de projet.

Mais c'est au tournant des années 1990 que l'UE commence à s'intéresser à ce mode particulier de gestion des 
territoires ${ }^{1}$. L'intégration de nouveaux pays et la construction de politiques à l'échelle communautaire créent alors des conditions favorables à la circulation d'idées, de nouveaux modèles et d'instruments politiques. La quête de solutions nouvelles pour le développement devient alors d'autant plus pressante que la perspective d'adhésion de l'Espagne et du Portugal pose clairement deux problèmes cruciaux à la communauté européenne des Dix : celui de la reconversion des industries traditionnelles concurrencées par ces nouveaux entrants disposant d'une main-d'œuvre peu coûteuse d'une part; et celui du freinage de l'exode rural et du niveau irrémédiable de dépeuplement atteint par certaines régions d'autre part (Jean, Baudelle, 2009).

Les décideurs politiques et administratifs européens comprennent alors vite le bénéfice qu'ils peuvent tirer du démontage du mécano du développement local. Ils vont alors pouvoir proposer, à quelques territoires se sentant délaissés ou menacés par le progrès de la construction européenne, de nouveaux outils pour prendre en main leur développement : en majorité des zones en reconversion industrielle et en déclin rural. L'aspect novateur de la démarche explique d'une certaine mesure que l'appui au développement local ait principalement pris au début la forme d'une myriade de programmes à caractère plus ou moins expérimental : actions innovatrices, projets pilotes, programmes d'initiative communautaire, etc. Dans un premier temps, fortement associé à la cohésion économique et sociale, le soutien au développement local s'est ensuite focalisé

1. Auparavant, le soutien au développement local se réalisait exclusivement dans une logique plutôt sectorielle à travers des Programmes d'initiative communautaire. L'action locale par le bas était encore en partie délaissée dans un contexte où les projecteurs médiatiques étaient braqués sur la compétitivité pour relever les nouveaux défis de l'économie mondialisée. sur la lutte contre le chômage et plus tard sur la cohésion sociale.

Mais c'est sous la forme d'une liste d'objectifs assignés aux mesures de soutien au développement local dans le cadre de la politique de cohésion, que la Commission a explicité pour la première fois, en 1991 (CE 240/1990), ce qu'elle attendait du développement local (Capron, 2006). Il devait permettre, entre autres, de sensibiliser les décideurs à l'importance des investissements doux et des facteurs immatériels dans le processus de développement territorial et ainsi rééquilibrer la prééminence des infrastructures lourdes dans les interventions européennes. Le développement local devait également permettre d'émanciper les populations sur le terrain en leur donnant des méthodes pour prendre en mains leur propre avenir et les aider à participer pleinement au mouvement d'intégration européenne. Enfin, il devait permettre d'accélérer l'apprentissage du développement durable au plus bas niveau de gouvernance, en misant sur une diffusion par imitation à d'autres territoires similaires et aux niveaux de gouvernance supérieurs (provincial ou département, régional et national).

Dès 2003, l'UE retient, dans ses conclusions du $4^{\mathrm{e}}$ rapport de cohésion (Commission européenne, 2004), le développement local comme l'une des modalités possibles d'utilisation des fonds structurels afin de renforcer la cohésion dans certains territoires à l'écart de la dynamique des centres et contribuer aux objectifs de la stratégie Europe 2020 (Commission européenne, 2004). Le programme pilote Leader est directement incorporé à la politique agricole de l'UE. Perçu comme une nouvelle façon de faire de la politique ascendante, à la base et sur des enjeux partagés par les acteurs locaux, il fait dès lors partie intégrante de la panoplie des instruments de l'action publique rurale, second pilier de la Politique agricole commune. 
En tant que projet politique, cette démarche Leader consiste à orienter les efforts de développement sur des projets conçus à l'échelle de communautés locales dans le but de renforcer les capacités d'initiatives et d'action des acteurs locaux selon une approche bottom-up (Commission européenne, 2006). Les décisions relatives à la stratégie de développement local ainsi qu'aux projets sont prises par des organes situés au plus près des périmètres d'intervention. Cette initiative politique, fondée sur l'hypothèse du développement endogène, est conçue sur un principe territorial et non une approche sectorielle. Son objectif est de valoriser des ressources locales de manière à maximiser le produit des activités et à en retenir les bénéfices, mais aussi de développer de manière ciblée les besoins, les capacités et les attentes de la population locale. L'initiative Leader doit également permettre la participation des acteurs locaux à la conception de leur propre stratégie de développement et sa mise en œuvre, et soutenir la recherche d'une maîtrise des conditions et des effets $\mathrm{du}$ développement par les populations concernées (expérimentation d'une démocratie participative) (Deberre, 2007).

Les nombreux travaux qui y sont consacrés mettent l'accent sur son caractère innovant et y voient une nouvelle étape du développement des zones rurales en Europe (Osti, 2000). Pour eux, l'originalité de Leader réside avant tout dans la méthode sur laquelle reposent la prise de décision et les actions qui en découlent. Dans une démarche de nature ascendante, la méthode Leader s'appuie sur les principes de subsidiarité et de partenariat. Dans le respect du principe de subsidiarité, l'UE a confié aux autorités nationales de chaque État membre le soin de définir le dispositif de mise en ouvre de l'axe Leader qui s'adresse aux acteurs locaux constitués en partenariat. Ce principe de partenariat implique la participation de ces acteurs, publics et privés, à la conception de la stratégie de développement et à son application. Celui-ci sous-tend la création d'une entité décisionnelle, un Groupe d'action locale (GAL) conçu comme maître d'œuvre des projets portés par ces mêmes acteurs (collectivités locales, entrepreneurs, exploitants agricoles, membres d'association, etc.) sur un territoire défini. En conférant au GAL un statut d'acteur collectif, la démarche Leader se présente sous le jour d'une décentralisation de l'action publique. Elle implique l'ouverture de nouveaux espaces de régulation entre de multiples catégories d'acteurs participant d'une gouvernance multiniveaux (européen, national, local).

À la logique antérieure d'une politique de développement déterminée et impulsée par le haut, il substitue le discours du développement autonome, la communauté locale devenant la source de légitimité (Bruckmeier, 2002). Dès lors, l'analyse des conditions de formation des GAL, l'examen de leur composition et de leur mode de fonctionnement s'avère fondamental pour comprendre la portée réelle de l'expérimentation du programme Leader. Parce que le modèle Leader comporte une dimension territoriale affirmée, il alimente un discours le décrivant comme un mode d'action plus efficace qu'une intervention de type exogène (Valqui-Vidal, 2009). On se plaît alors à souligner la dimension humaine de l'outil Leader étant donné la place accordée à la participation de la communauté locale dans la recherche d'une maîtrise des conditions et des effets du développement.

\section{Une traduction territoriale différenciée des modalités d'application du programme Leader}

Replacées dans le contexte et les réformes institutionnelles propres à chaque État, les différentes modalités de transpositions nationales guidant la mise en œuvre du 
programme Leader expliquent en partie la diversité des formes que peut prendre l'action locale (Berriet-Solliec Trouvé, 2010).

\section{Un maillage de GAL de forme hétérogène soumis aux structures institutionnelles}

D'une programmation Leader à l'autre, le maillage des GAL s'est progressivement densifié. Cette extension, liée pour partie aux changements de règles d'éligibilité qui ont tendance à densifier le maillage, semble témoigner d'une meilleure intégration de l'ensemble des campagnes dans les dispositifs d'aménagement des politiques d'intervention. En France, par exemple, le conditionnement de création d'un GAL à l'existence de territoires déjà organisés (notamment des pays) dont le nombre s'est fortement accru ces dix dernières années explique pour partie cette densification.

Aujourd'hui, plus de 1800 régions Leader couvrent près de $40 \%$ de la superficie totale des espaces ruraux européens et concernent plus de $60 \%$ de la population rurale. Pour la période 2007-2012, chaque pays en a retenu un nombre plus ou moins important dont la taille varie amplement selon le contexte local ou national (figure 1).

C'est en Allemagne et en France que le nombre de GAL est le plus important (avec respectivement 243 et 300 régions Leader) ; mais de taille relativement modeste, ils ne concernent environ que $60 \%$ de la population rurale totale. En France, le nombre de GAL est passé de 140 dans le

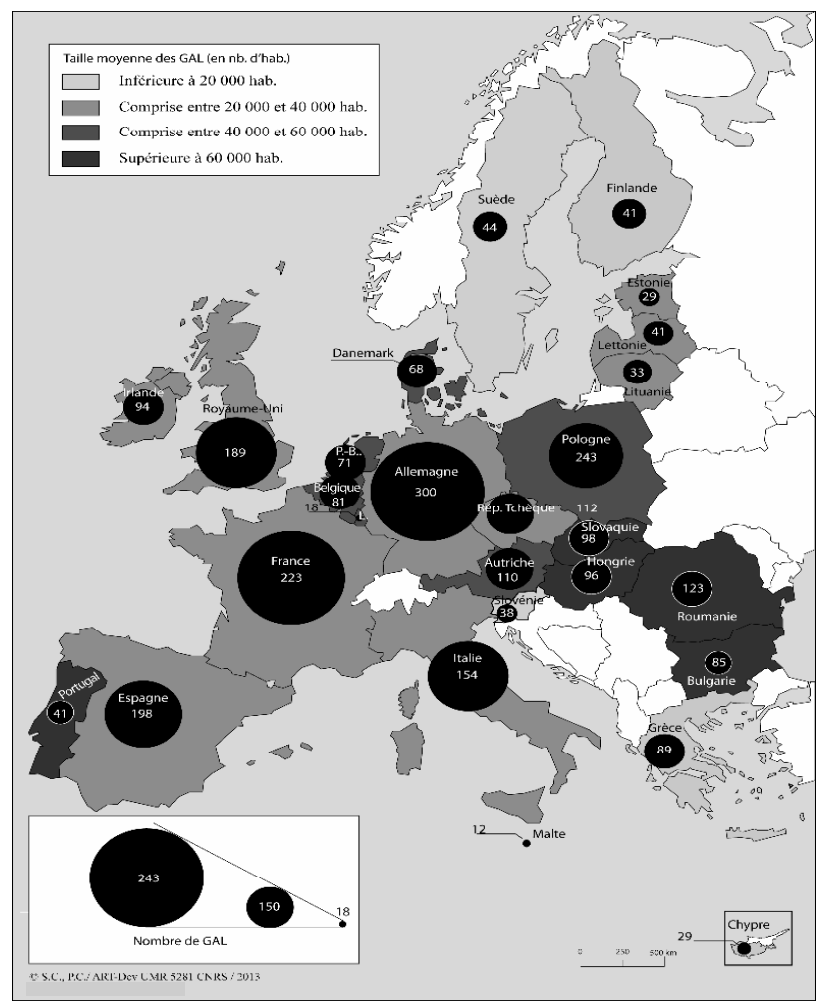

Source : Commission européenne. 
RECHERCHES

Figure 2. Écart-type entre la taille minimale et la taille maximale des GAL en 2012 (en nombre d'hab.)

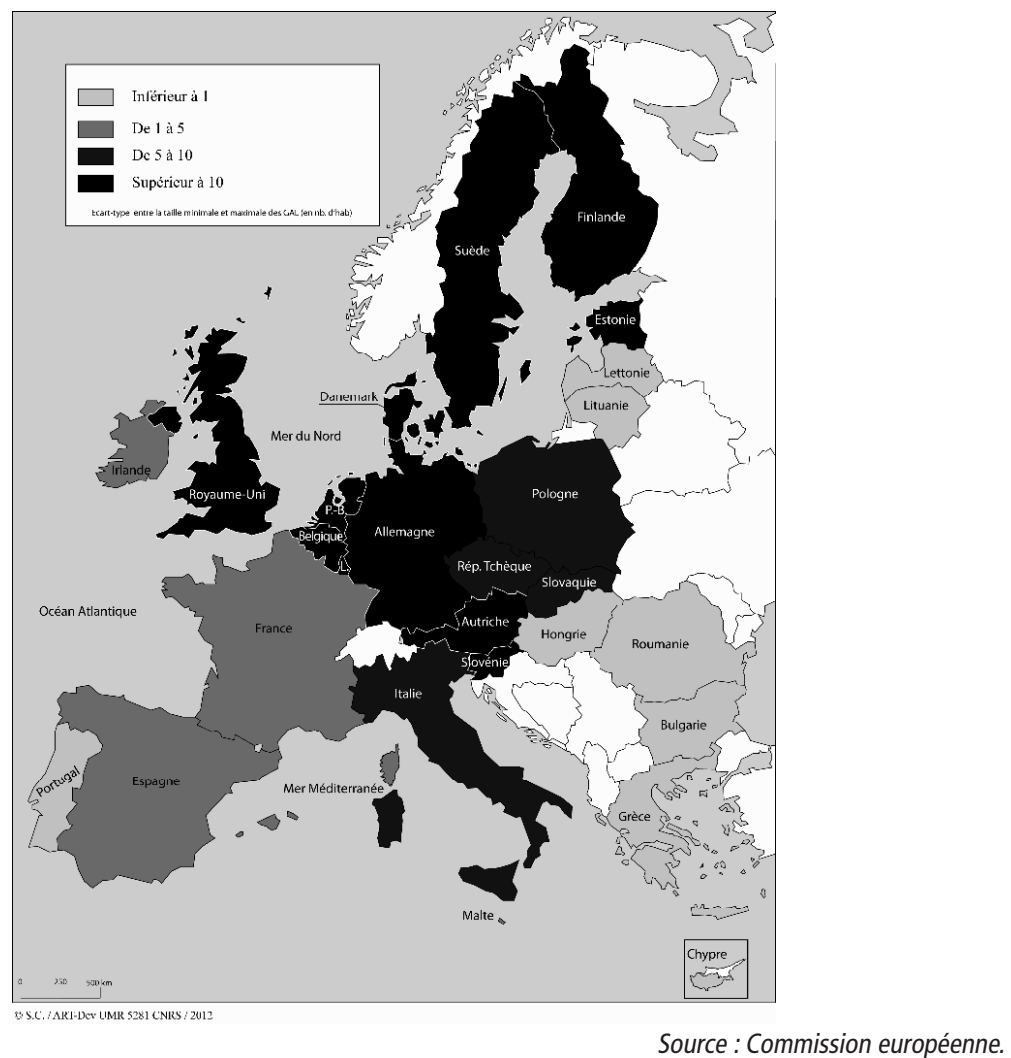

cadre du programme Leader + à $223 \mathrm{GAL}$ dans la programmation actuelle. Les GAL ne cessent de gagner du terrain occupant désormais, en termes de superficie, près de la moitié de l'espace rural français. Leader 2007/2013 accueille dans ses GAL 7584 communes n'ayant pas participé précédemment au programme Leader + . Douze millions d'habitants y sont intégrés (soit plus de $70 \%$ de la population rurale nationale). Mais c'est dans les nouveaux États membres d'Europe centrale que la taille des GAL est la plus importante. En Hongrie, en Bulgarie et en Roumanie, les GAL sont en moyenne deux fois plus peuplés que dans les autres pays de l'UE. La couverture du territoire national par le réseau des GAL y est quasi exhaustive comme en Lettonie et en Hongrie où elle atteint $88 \%$ de la superficie rurale totale. Cette couverture y est une fois et demie plus étendue que la moyenne européenne et trois fois plus que celle des pays scandinaves et du Royaume-Uni où le maillage irrégulier des GAL se présente comme une mosaïque parsemée d'espaces en blanc, les communes restées à l'écart du processus (Chevalier, Dedeire, Pola, Kovacs, 2012).

Les caractéristiques de taille sont parfois hétérogènes à l'intérieur même d'un pays. En Allemagne, la taille des Gal peut varier amplement de 1 et 13 (figure 2). Au Royaume-Uni, en Belgique, aux Pays-Bas et en Estonie, où les périmètres d'action procèdent en général du libre choix des acteurs locaux pour construire leur propre territoire, la taille des GAL peut varier, dans une même région, d'une dizaine de 
milliers d'habitants à plus de 150 000. En revanche, l'écart-type est bien plus faible en Grèce et en Lituanie où la taille des régions Leader est relativement homogène. Dans ces pays, la Commission européenne note d'ailleurs « une tendance à vouloir unifier la taille des régions Leader » (Commission CE-2011-8). Dans ce cas, les périmètres des régions Leader peuvent s'inscrire dans une volonté de consolidation, voire de légitimation de périmètres institutionnels existants dont la taille équivalente permet une généralement meilleure gestion du territoire (dans le cadre d'une politique d'aménagement régional). Mais est-ce vraiment l'objectif du développement local de soutenir et de participer à la gestion des territoires institutionnels ?

D'une manière générale, on peut se demander d'où proviennent de telles différenciations ? Certes, la géographie des régions Leader peut traduire les discontinuités de peuplement qui expriment la diversité des campagnes européennes, les GAL semblant moins nombreux, moins peuplés et plus étendus dans les régions de très faibles densités scandinaves que dans une Europe centrale relativement peuplée. Mais les facteurs explicatifs d'une telle diversité sont plus complexes à cerner.

La diversité des situations socio-économiques pourrait expliquer l'absence, ou au contraire, la démultiplication des régions Leader. La prospérité agricole tendrait à exclure toute forme de développement alternatif dans un contexte économique dominé par la rente agricole, la performance et l'intégration agro-industrielle des grandes exploitations. À l'inverse, la faible intégration économique inciterait à trouver des voies alternatives au modèle dominant par des projets plus innovants. La dynamique de création des GAL refléterait également la capacité des acteurs locaux à initier des actions collectives. Dans certaines régions, alors que la destruction des sociétés locales, le chômage, l'émigration des forces vives et le vieillissement inhiberaient les initiatives locales dans la conception de projets de développement, dans d'autres, la revitalisation du tissu social et économique doperait les initiatives locales.

Mais, si l'on y regarde de plus près, la situation socio-économique ne semble pas déterminante a priori. En Allemagne, dans les grandes plaines agricoles du centre et du nord du pays, malgré la performance et l'intégration des économies rurales aux grands marchés agricoles internationaux, le nombre de GAL a augmenté depuis les précédentes programmations Leader (Lacquement, 2008). En France, plus de la moitié des territoires de projet actuels, quelle que soit leur localisation, mettent en œuvre une approche Leader pour la première fois. En Bulgarie et en Roumanie, la crise socio-économique profonde ne semble pas non plus avoir affecté la dynamique de création des régions Leader. En Hongrie, en Baranya et dans l'extrême Est du pays, le nombre de GAL a même été multiplié par deux par rapport aux premières initiatives Leader + en 2004 alors que l'exode rural, le vieillissement des populations et la crise économique se sont fortement accentués ces dernières années.

En fait, la dynamique des programmes Leader semble moins corrélée aux dynamiques socio-économiques locales qu'à la volonté politique forte de promouvoir plus ou moins intensément les démarches de développement endogène - d'autant que la démarche Leader est désormais conçue comme une mesure d'accompagnement intégrée à chaque politique nationale de développement rural. Certes, les modalités de transformation socio-économique des campagnes - en modifiant la structuration des sociétés locales - ont fait émerger de nouveaux acteurs susceptibles de porter des initiatives locales ; mais ce sont surtout les modalités de gestion et de mise en ouvre par les acteurs institutionnels du programme introduit par la logique 
de transfert institutionnel - qui semblent refléter la géographie des régions Leader ; les acteurs institutionnels influent largement sur la dynamique et la forme que prennent les territoires d'action locale.

\section{Un effet de structuration des territoires par les périmètres institutionnels}

En fait, le rapport de la commission européenne en décembre 2011 (CE 2011-08) conclut que seulement 10 à $20 \%$ des périmètres actuels des territoires Leader suivent globalement les contours « d'une unité physique, historique ou culturelle identifiée et homogène ». Essentiellement en Allemagne, en Suède ou en Finlande, cette construction des périmètres par la recherche d'une région homogène identifiable par son paysage ou son histoire remplit d'autant mieux les critères d'éligibilité qu'elle semble en partie s'appuyer sur un ensemble de communes généralement liées par des relations économiques et une histoire commune. Mais de manière générale, le maillage des régions Leader semble, dans la majorité des cas, être soumis à l'effet de tropisme qu'exercent les structures institutionnelles et/ou les périmètres d'intervention préexistants. En France, $69 \%$ des GAL sont calqués sur les périmètres des Pays (loi Voynet) ou des Parcs naturels régionaux ${ }^{2}$. La superposition des limites des Pays sur les périmètres des GAL révèle l'effet structurant des Pays sur la composition des GAL. Certes, ces entités ne sont pas des territoires institutionnels au sens juridique du terme, mais leur construction, aussi endogène puisse-telle paraître, s'appuie désormais en grande partie sur l'adhésion des communautés de communes (création institutionnelle par essence) qui tendent à se substituer aux communes comme unité locale de base.

2. La législation impose d'ailleurs aujourd'hui de faire coïncider les maillages des pays et des Parc naturels régionaux (PNR) avec ceux des régions Leader (RDR, 2007).
En Espagne, en Allemagne, en République tchèque et en Italie, dans le cadre de « l'articulation avec les politiques régionales », les projets d'aménagement associent souvent étroitement les groupes Leader au cofinancement d'une série d'actions prévues par les chartes de développement des territoires de projet déjà existants (microrégions, comarcas, etc.). À ce titre, la plus ou moins grande facilité d'obtenir un cofinancement joue d'ailleurs un rôle discriminant dans la réussite ou l'échec d'un projet de territoire. Ainsi, très souvent, la superposition des périmètres d'intervention se fonde sur le recoupement des réseaux d'acteurs qui sont impliqués dans les deux dispositifs. Certes, elle favorise alors souvent la concentration des investissements, facilite la gestion et le montage des projets et, probablement, accélère la réalisation des projets. Mais, cette superposition confine très souvent les GAL dans une fonction supplétive d'une politique d'intervention décidée et gérée par les États eux-mêmes.

Bien évidemment, les périmètres des territoires de projet, dans l'immense majorité des cas, ne se superposent pas physiquement pas aux maillages d'aménagement ou de gestion existants (régions, départements, etc.). Ils les englobent parfois, les chevauchent, voire les recoupent. Néanmoins, les contraintes de types administratives qui en découlent sont souvent de nature à jouer un rôle de frein dans la dynamique du territoire de projet (démultiplication des dossiers de demande de subvention en fonction du nombre de régions partenaires des GAL en France, obligation de déposer les projets de GAL dans chaque comarcas intégrée au programme Leader espagnol, etc.).

Mais parfois le principe d'autonomisation du processus de création et de gestion des GAL qui sous-tend le principe même de gouvernance locale, est plus directement remis en question lorsque les contours des 
régions Leader suivent les limites des circonscriptions administratives du niveau local, voire les circonscriptions électorales. La coïncidence peut être parfaite ou imparfaite lorsque le périmètre de la région Leader contourne simplement les communes les plus urbanisées, non éligibles au programme européen. Au Portugal, comme en Hongrie, la volonté de mettre en place de nouveaux échelons institutionnels avec la généralisation de la régionalisation a vraisemblablement perturbé les processus spontanés de regroupement intercommunal. En Slovaquie ou en Lituanie, la volonté de caler les territoires des GAL sur les collectivités microrégionales et les districts a également fortement contraint la construction des partenariats. En Bulgarie et en Roumanie, on a le sentiment que le dispositif Leader sert d'appui à des interventions gérées par l'administration locale dans des circonscriptions dont l'extension a été modifiée par les réformes successives depuis 1990 de manière à gérer et organiser les services et les équipements publics. Globalement, quel que soit le contexte national, la satellisation administrative des périmètres d'intervention des programmes Leader tend alors à confirmer la faiblesse du potentiel d'autonomisation de l'initiative locale et une certaine formalisation du développement endogène. En Hongrie, les limites des GAL correspondent avec celles des microrégions (réunies par deux ou par trois) et épousent assez fidèlement le découpage en circonscriptions électorales. En fonction de l'appartenance politique des élus locaux, la formation des périmètres Leader a obéi à une logique partisane, les affinités clientélistes l'emportant sur le souci de renforcer la cohésion territoriale.

\section{Un cadre institutionnel entravant la démarche de développement local ?}

L'action européenne de développement local peut être entendue comme une politique de soutien au développement qui prend appui sur la constitution d'un territoire fondé sur un projet. Elle s'insère dans le mouvement de reterritorialisation de l'action publique promu par l'UE qui doit répondre, en théorie, à une définition plus localisée des problèmes publics et des moyens de leur prise en charge. Dans la philosophie européenne du développement local, il s'agit d'une politique territoriale qui privilégie la participation des acteurs et les initiatives émanant de la base ; autrement dit, elle est par nature propre à un territoire librement construit par les acteurs locaux «par et pour un projet ». Mais dans la pratique, intégrée (voire encastrée) dans son cadre réglementaire, la démarche Leader ne devient-elle pas une simple politique territorialisée, c'est-à-dire une forme de régulation territoriale par les États qui la mettent en œuvre? (Lascoumes, Le Gales, 2007). Et dans ce cadre, que reste-t-il des principes de base du développement local ?

Sur le plan réglementaire, des logiques contradictoires sous-tendent la forme de l'action publique Leader. En créant les conditions de prise en charge du développement des territoires locaux par l'Europe et les États, elle est en fait le vecteur d'une diffusion de nouvelles normes, parfois « très contraignantes », qui encadrent la mise en œuvre du développement local. L'application de procédures relativement strictes, venues essentiellement des États, quelquefois couplée à des phénomènes de mimétisme propagés par les consultants ou groupes d'experts et les logiques inhérentes à la généralisation des cofinancements sont à l'origine d'une certaine standardisation du développement local qui contrecarre l'idée d'une définition localisée des problèmes publics et de leur prise en charge. Parfois, «l'importance des jeux de contrôle territorial par les instances nationales tend à reproduire un ordre territorial où l'action publique prend forme dans des espaces qui sont bornés a priori [...]. La défense du territoire institutionnel devient alors la logique d'action dominante 
RECHERCHES

des États au risque d'inhiber les initiatives locales » (Maurel, 2008). Dès lors, il est intéressant de confronter le discours européen sur la territorialisation de l'action publique - qui privilégie l'approche bottomup dans la mise en œuvre de la politique de développement rural - à sa traduction effective dans les États.

\section{Un degré de sélectivité des GAL symptomatique d'une institutionnalisation du programme ?}

La démarche Leader participe de cette dynamique politique qui consiste à promouvoir le développement socio-économique des zones rurales par des initiatives locales ciblées et une concentration des moyens. D'après la Commission européenne (CE, 2008-07), la mise en place de procédures sélectives des GAL doit permettre de financer des projets et d'éviter une dispersion des budgets. Mais est-ce vraiment le cas ? Comment est opéré le choix des GAL ? Et comment les budgets sontils répartis ? C'est à partir de la notion de sélectivité, définie comme la capacité des pays à concentrer le nombre de GAL et le financement sur des projets, que l'on peut en partie répondre à cette question.

\section{Figure 3. Indice de sélectivité}

Pour évaluer le degré de concentration des financements et du nombre de GAL nous avons calculé un indice de sélectivité construit sur la base du croisement des deux variables suivantes :

- le nombre de GAL sélectionné par pays par rapport au nombre de projets déposés.

- le montant financier moyen attribué par GAL par l'organisme gestionnaire par rapport au budget prévisionnel demandé.

La somme des résultats de ces deux rapports, est ensuite codée de manière arithmétique simple, sur une base 100 par rapport à une valeur médiane calculée à l'échelle européenne. Plus une valeur est négative, plus le pays est considéré comme sélectif dans ses choix. Le rapport entre le nombre de projets sélectionnés à ceux déposés est inférieur à la moyenne européenne, tout comme le rapport entre le budget accordé à chaque GAL et celui demandé. A l'inverse, plus la valeur est positive, moins le pays est sélectif dans ses choix.

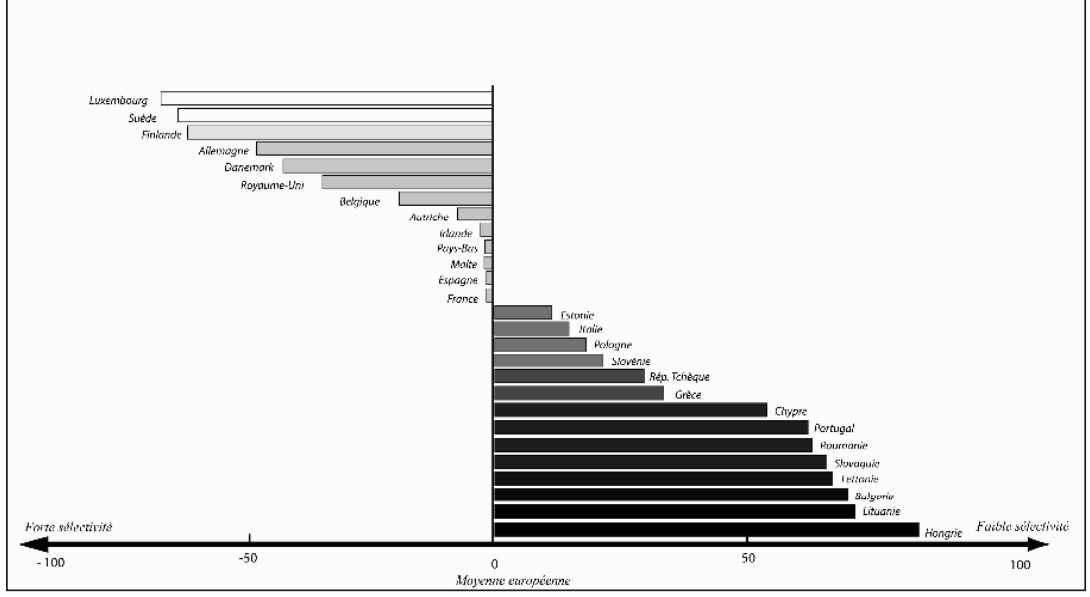

Sources : Instituts statistiques nationaux. 


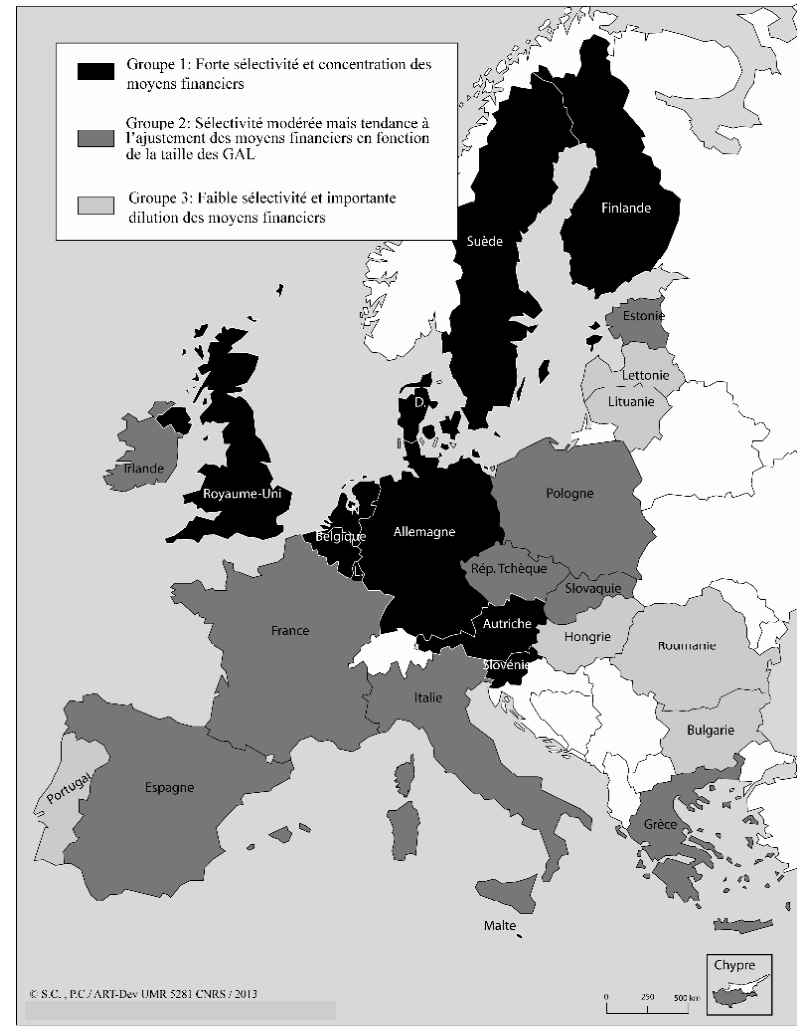

Sources : Commission européenne / Instituts statistiques nationaux.

L'indice de sélectivité (figure 3), croisé avec les caractéristiques nationales des GAL permet d'aboutir à une typologie $(f i-$ gure 4) qui apporte une information sur les logiques qui ont présidé à la mise en œuvre de l'axe Leader dans chaque pays.

Dans les pays d'Europe médiane (groupe 1$)^{3}$, la sélectivité semble avoir

3. Groupe 1 :

- Indice de sélectivité supérieur à la moyenne européenne ;

- Écart-type taille minimale/taille maximale des GAL supérieur à la moyenne européenne ;

- Part de la superficie couverte par les GAL inférieure à la moyenne européenne ;

- Écart-type de la distribution des budgets entre les GAL supérieure à la moyenne européenne ;

- Coefficient de corrélation entre le budget moyen des GAL et le nombre d'habitants inférieur à 0.1 . été relativement soutenue. Globalement, $40 \%$ des projets de GAL ont été rejetés par l'autorité de gestion et la répartition du budget, très inégale selon les territoires, a peu tenu compte de la taille des régions Leader (faible coefficient de corrélation entre la taille des GAL et leur budget). En Allemagne, dans les pays scandinaves et au Royaume-Uni, l'indice de sélectivité est globalement huit fois supérieur à la moyenne européenne. En Finlande, sur 59 dossiers proposés, seuls $31 \mathrm{GAL}$ ont été sélectionnés. Dans ces pays, la compétition, fondée sur la qualité de montage des dossiers, a permis de sélectionner un nombre élevé de GAL dont les caractéristiques de taille et de définition du périmètre sont généralement très hétérogènes. 
De l'Irlande à l'Allemagne et de l'Italie à la Finlande, les périmètres d'action procèdent généralement du libre choix des acteurs locaux pour construire un territoire pertinent, doté de cohérence et disposant généralement des potentialités suffisantes pour justifier d'un projet.

L'importance et la qualité des relations sociales dans les territoires et parfois l'existence de coopérations antérieures ont d'ailleurs très souvent facilité le processus de rapprochement des communes et l'élaboration des périmètres des GAL.

Loin de montrer un territoire couvert en totalité par un maillage régulier, la carte des GAL dans ces pays se présente comme une mosaïque d'espaces restés à l'écart du processus. Ce mode de formation des territoires de projet semble plus en accord avec la logique ascendante qui caractérise, en théorie, la création des territoires de projet.

Dans le groupe $2^{4}$, la situation est moins tranchée. L'indice de sélectivité y est conforme à la moyenne européenne. Certes, le nombre de GAL retenu par l'autorité de gestion est inférieur au nombre de candidatures déposées, mais le budget alloué par GAL à tendance à être corrélé à la taille des régions Leader. En France, où la procédure est régionalisée, le rapport de l'évaluation de la sélection des candidatures Leader 2007-2013 (ministère de l'Agriculture/Agence de services et de paiement, mars 2010) conclut que les procédures ne sont pas toutes en adéquation avec le principe du modèle européen

4. Groupe 2 :

- Indice de sélectivité supérieur égal à la moyenne européenne ;

- Écart-type taille minimale/taille maximale des GAL égale à la moyenne européenne ;

- Part de la superficie couverte par les GAL égale à la moyenne européenne ;

- Écart-type de la distribution des budgets entre les GAL égale à la moyenne européenne ;

- Coefficient de corrélation entre le budget moyen des GAL et le nombre d'habitants inférieur à 0.5 . de développement, et n'ont pas réussi à concentrer les financements Fonds européen agricole pour le développement rural (FEADER), les recommandations financières étant moins bien suivies notamment dans les petites régions. Celles-ci ont souvent ajouté des contraintes supplémentaires quand à la répartition de leur budget en incitant les GAL à justifier leur demande, non pas en fonction de leur propre stratégie de développement locale, mais de la moyenne de l'assiette fiscale de l'ensemble des communes membres. En Estonie, l'attribution du budget des GAL, prenant en compte l'innovation et l'originalité de la stratégie locale, est pondérée en fonction du revenu moyen des habitants vivant dans la région Leader. La logique de redistribution des fonds aux territoires en retard de développement est-elle conforme aux orientations du modèle européen de développement local qui prônent avant tout la compétition et l'innovation?

Enfin, l'analyse statistique identifie un troisième groupe de pays ${ }^{5}$, dont la sélectivité est la plus faible. En général, le nombre de GAL retenu par l'autorité de gestion est sensiblement égal à celui des candidatures déposées. Dans ces pays, les opérateurs de transfert semblent avoir privilégié la gestion (en renforçant la réglementation) au détriment de la compétition et de l'innovation. La sélection des projets, théoriquement définis autour de critères de qualité du programme et du partenariat, a mal fonctionné ; tous les projets déposés ayant été retenus. En général, dans une

5. Groupe 3 :

- Indice de sélectivité supérieur inférieur à la moyenne européenne ;

- Écart-type taille minimale/taille maximale des GAL inférieur à la moyenne européenne ;

- Part de la superficie couverte par les GAL supérieur à la moyenne européenne ;

- Écart-type de la distribution des budgets entre les GAL inférieur à la moyenne européenne ;

- Coefficient de corrélation entre le budget moyen des GAL et le nombre d'habitants supérieur à 0.8 . 
perspective de distribution égalitaire des fonds, la répartition du budget est relativement homogène. Elle est également souvent fonction du nombre d'habitants inclus dans la région Leader (fort coefficient de corrélation entre le nombre d'habitant et le budget du GAL). La couverture du territoire national par les GAL est quasi exhaustive. Les GAL, relativement identiques, sont de taille plus grande et sont en moyenne deux fois plus peuplés qu'ailleurs en Europe. Sous la forme d'un maillage homogène de l'espace rural, ils deviennent le cadre d'application de la politique de développement rural, dans une simple logique de redistribution des aides au développement ${ }^{6}$. C'est le cas du Portugal, qui par souci de non-exclusion d'une partie de son territoire aux aides européennes (mais aussi sans doute par souci électoral) semble faire du programme Leader une simple politique de guichet. $\mathrm{Ce}$ pays a défendu le fait de retenir tous les projets déposés, quelle que soit la qualité de ceux-ci. Malgré un avis négatif sur certains projets par le groupe d'experts nationaux au regard des critères objectifs établis à partir d'une grille de sélection nationale, tous les dossiers ont in fine été retenus à la suite de l'avis du Conseil d'État. Les élus ont préféré diminuer l'enveloppe de chacun des projets pour faire en sorte que tous en bénéficient. Cet exemple illustre bien les contradictions d'une politique de projet se rapprochant, à terme, dans sa traduction concrète, d'une logique de guichet.

$$
\begin{aligned}
& * \\
& * *
\end{aligned}
$$

$\mathrm{Au}$ terme de ce travail, il est possible d'identifier des distorsions entre les modalités de mise en œuvre du programme Leader et les principes de base

6. L'écart-type de la distribution des budgets est de 0,12 et le montant des budgets est corrélé avec la taille des $\operatorname{GAL}(0,86)$. du développement local qu'il est pourtant susceptible de porter. Globalement, quel que soit le pays, le mode de composition des GAL peut être interprété comme la traduction d'une sorte de formalisation de la démarche Leader et finalement comme un encadrement de l'initiative locale par des normes de plus en plus prescriptives établies aux niveaux supérieurs du système territorial. Cette composition doit former un ensemble équilibré et représentatif des partenaires issus, d'une part des élus et de l'Administration et, d'autre part, des différents milieux socio-économiques du territoire local afin d'exprimer et de mettre en pratique les principes de la gouvernance locale comme nouveau mode de gestion des territoires ruraux.

Au début de la mise en œuvre du programme Leader dans les années 1990, on a eu le sentiment que la société civile, émancipée par les différents processus de décentralisation à l'Ouest et le retour à la démocratie à l'Est, était capable de prendre seule des initiatives propices au développement rural, indépendamment des pouvoirs politiques et des organisations économiques dominantes. Mais très vite, surtout à partir de la campagne Leader II (1994-1999), la contractualisation des programmes Leader a progressivement mis sous tutelle les GAL dont la composition est, depuis, devenue quasiment invariable : sont ainsi représentés dans tous les pays, les représentants des communes et des institutions appartenant au périmètre d'intervention, les principaux organismes professionnels des secteurs agricole, touristique et artisanal, les grandes organisations syndicales, notamment dans l'agriculture, les institutions de sauvegarde du patrimoine, de protection de l'environnement et des paysages, enfin quelques entreprises et un certain nombre d'associations sportives ou culturelles. D'une campagne à l'autre, d'un dispositif à l'autre, les prescriptions se font plus ou moins directives 
et finissent souvent par contraindre les principaux acteurs dans la construction des GAL à composer le groupe de pilotage de manière presque mécanique en sollicitant les acteurs locaux simplement en fonction de leur situation professionnelle ou de par leur niveau de responsabilité administrative dans l'intention d'établir les équilibres de représentativité finalement exigés par les organes décisionnaires de l'opérateur de transfert. Cette dimension prescriptive de la contractualisation, qui s'inspire des principes mêmes de la nouvelle gouvernance (Callahan, 2007) et les a établis en critères d'éligibilité, a figé la composition des GAL en faveur du développement endogène. Ces derniers semblent produire une sorte de standardisation de la représentation de la société locale. Ce cadre imposé par les opérateurs de transfert peut-il alors encore refléter véritablement la réalité sociale comme le prône le modèle européen ?

La désignation et le rôle du responsable du GAL par certaines administrations reflètent encore plus ce processus généralisé de formalisation du développement local. En Espagne, près du tiers sont pilotés par des membres ou des employés de l'Administration, principalement les autonomies et les comarcas. En Hongrie, en République tchèque et en Bulgarie, plus de $90 \%$ des GALS sont dirigés par des Maires. En outre, le poids des héritages agraires se traduit aussi, dans quelques cas (notamment les grandes régions agricoles allemandes et du nord de l'Italie) par l'intervention du syndicat agricole majoritaire, qui représente en réalité les intérêts des grandes exploitations. Les responsables issus du monde de l'entreprise sont, quant à eux, minoritaires. Ceci dit, lorsqu'ils existent, particulièrement au Royaume-Uni, aux Pays-Bas et au Danemark, ils développent essentiellement des projets ciblés autour de leur activité et notamment de la valorisation commerciale de leurs produits, par exemple dans le secteur agroalimentaire ou celui de la construction. Pour le reste des GAL, leur responsable peut être désigné par une structure associative qui tend en général à épouser les relations institutionnelles déjà nouées à l'échelle locale. Notons que cette typologie varie selon les pays : la gestion administrative et politique domine dans les anciens pays d'Europe centrale et orientale. Et alors que les structures associatives sont largement majoritaires en Scandinavie, en Italie et en Irlande, le milieu professionnel agricole est très présent en Italie et en Espagne et se distingue par l'implication de l'initiative privée. Ces différences tiennent sans doute aux nuances législatives de chaque pays et aux orientations de la politique de développement rural retenue par chacun des États. Elles reflètent néanmoins aussi, dans certaines régions (notamment en Europe centrale), la faiblesse des tissus économiques locaux et la fragilité des sociétés locales, lesquelles justifient que l'initiative endogène soit encadrée et que l'apprentissage de la gouvernance locale soit dirigé par les autorités administratives ou soit prise en charge par des structures dans la dépendante de ces dernières. Cela n'est pas sans conséquence pour la formation et le fonctionnement des réseaux d'acteurs qui portent les projets de développement.

L'animateur d'un GAL est officiellement le coordinateur de l'action de développement et se situe à l'interface entre intérêts publics et privés. Dans les faits, il semble monopoliser la «participation locale » autour du groupe constitué qui finit par former une « sorte de classe de projet » (Kovach, 2002) parce que ses membres sont les rares acteurs de la société locale à posséder les compétences, les prérogatives ou les capitaux et à pouvoir les mettre au service du projet de développement. D'ailleurs, les membres des GAL sont presque toujours dans les autres dispositifs d'aménagement à l'échelle locale ou régionale (Parcs naturels, communautés de 


\section{Application du programme Leader selon le développement local}

communes, microrégions, Pays, etc.). La logique de réseau s'avère alors parfaitement opérationnelle : en sollicitant tous les « guichets » disponibles et en usant de tous les ressorts de la contractualisation avec les différents échelons de l'administration, elle permet de multiplier les actions de développement et de compléter le financement des projets. Toutefois, dans le même temps, d'une campagne Leader à l'autre, elle tend à confisquer l'initiative endogène au profit d'acteurs choisis qui finissent par devenir les relais locaux des orientations décidées aux niveaux supérieurs du pouvoir politique. Les diagnostics de territoire qui composent les dossiers de candidature au programme Leader dans près de $80 \%$ des pays européens, sont très significatifs de ce point de vue : ils revêtent, pour la plupart, un caractère mimétique et normatif et ne visent finalement qu'à justifier l'éligibilité du territoire local à l'aide financière.

\section{RÉFÉRENCES BIBLIGRAPHIQUES}

Baudelle G., Guy C., Mérenne-Schoumaker B. (2011). Le développement territorial en Europe. Concepts, enjeux et débats. Rennes, Presses universitaires de Rennes, 288 p.

Berriet-Solliec M., Trouvé A. (2010). La politique agricole commune est-elle territoriale? In Les mondes agricoles en politique, Chapitre 13, Paris, Presses de Sciences Po, p. 397-413.

Bruckmeier K. (2002). Leader in Germany and the Discourse of Autonomous Regional Development. Sociologia Ruralis, vol. 40, $\mathrm{n}^{\circ} 2$, p. 219-227.

Buller H. (2000). Re-creating Rural Territories: LEADER in France. Sociologia Ruralis, vol. 40, n², p. 190-199.

Bulmer S., Dolowitz D., Humphereys P., Padgett S. (2007). Policy Transfer in European Union Governance: regulating the Utilities, Abingdon, Routledge, 94 p.

Callahan R. (2007). Governance: the collision of politics and cooperation. Public Administration Review, ${ }^{\circ}$ 67/2, p. 290-301.

Capron P. (dir.) (2006). Convergence et dynamique d'innovation au sein de l'espace européen. Bruxelles, De Boeck.

Chevalier P., Dedeire M., Pola P., Kovacs D. (2012). The implementation of the Leader program in central Europe: between a local development approach and political instrumentalization. Discussion Paper, $\mathrm{n}^{\circ} 89$, Académie des Sciences de Hongrie, 30 p.

Chevalier P., Jean Y. (2011). Les campagnes d'Europe occidentale : vers de nouvelles trajectoires de diversification ? In Jean Y., Guibert M. (dir.), Dynamique des espaces ruraux dans le monde, Paris, Armand Colin, collection Universitaire, p. 141-160.

Commission européenne (2001). Rapport de la commission au parlement européen, au conseil, au comité économique et social européen et au comité des régions concernant la mise en ouvre des plans stratégiques nationaux et orientations stratégiques de la communauté pour le développement rural (période de programmation 2007-2013. Com $52011^{\circ} 943$ final.

Commission européenne (2004). Investir dans l'avenir de l'Europe. Quatrième rapport sur la cohésion économique, sociale et territoriale, OPUE.

Commission of the European communities (1996). The Cork Declaration: a living countryside.

Commission européenne, Direction générale de l'agriculture et du développement rural (2006). Guide de base : l'approche LEADER. Fact Sheet, Office des publications officielles des Communautés européennes. 
RECHERCHES

Pascal CHEVALIER, Marc DEDEIRE

Commission européenne. (2007). Règlement de Développement Rural (RDR), (CE) $n^{\circ} 1698 / 2005$.

Deberre J.-C. (2007). Décentralisation et développement local. Afrique contemporaine, $\mathrm{n}^{\circ} 221$, p. 45-54.

Delpeuch T. (2009). Comprendre la circulation internationale des solutions d'action publique : panorama des Policy Transfer Studies. Critique internationale, $\mathrm{n}^{\circ} 43$, p. 153-166.

Dolowitz D., Marsh D. (2000). Learning from Abroad: The Role of Policy Transfert in Contemporany Policy Making. Governance, vol. $13, \mathrm{n}^{\circ} 1$, p. $5-24$.

Greffe X. (2005). La décentralisation. Paris, La Découverte, coll. « Repères », 122 p.

Houée P. (2001). Le développement local au défi de la mondialisation. Paris, L'Harmattan, $249 \mathrm{p}$.

Kovach I. (2002). Leadership, Local Power and Rural Restructuring in Hungary. In Halfacree K., Kovach I., Woodward (dir.), Leadership, Local Power and Rural Restructuring in Contemporary Europe, Aldershot, Burlington, Singapore, Sydney, Ashgate, p. 91-121.

Lacquement G. (2008). Du développement local dans les nouveaux Länder allemands : acteurs et territoires des programmes d'initiative communautaire. Revue d'études comparatives Est-Ouest, vol. 39, n ${ }^{\circ}$, p. 81-112.

Lascoumes P., Le Gales P. (2007). Sociologie de l'action publique. Paris, Armand Colin, $126 \mathrm{p}$.

Maurel M.-C. (2008). L'action publique «par le bas » : L'approche LEADER en Europe centrale. Revue d'études comparatives EstOuest, vol. 39, n 4, p. 33-61.

Muller P., Rouault S. (1997). Une grammaire européenne de l'expérimentation sociales. Cultures et conflits, $\mathrm{n}^{\circ} 28$, p. 75-97.

Osti G. (2000). LEADER and Partnerships: the case of Italy. Sociologia Ruralis, vol. 40, $\mathrm{n}^{\circ} 2$, p. 172-180.

Pecqueur B. (2000). Le développement local, $2^{\mathrm{e}}$ édition, Paris, Syros.

Smith A. (1995). L'Europe politique au miroir du local. Les fonds structurels et les zones rurales en France, en Espagne et au Royaume-Uni, Paris, L'Harmattan, 300 p.

Teisserenc P. (1994). La mobilisation des acteurs du développement local. Sociétés contemporaines, $\mathrm{n}^{\circ} 18 / 19$, Paris, l'Harmattan, p. 187-213.

Valqui-Vidal R.-D. (2009). Rural development within the EU LEADER+: new tools and technologies. AI \& Society, $\mathrm{n}^{\circ} 23$, p. $575-602$. 